MATHEMATICS OF COMPUTATION

Volume 69, Number 229, Pages 159-176

S 0025-5718(99)01131-X

Article electronically published on March 10, 1999

\title{
GLOBAL SUPERCONVERGENCE FOR MAXWELL'S EQUATIONS
}

\author{
QUN LIN AND NINGNING YAN
}

\begin{abstract}
In this paper, the global superconvergence is analysed on two schemes (a mixed finite element scheme and a finite element scheme) for Maxwell's equations in $R^{3}$. Such a supercovergence analysis is achieved by means of the technique of integral identity (which has been used in the supercovergence analysis for many other equations and schemes) on a rectangular mesh, and then are generalized into more general domains and problems with the variable coefficients. Besides being more direct, our analysis generalizes the results of Monk.
\end{abstract}

\section{INTRODUCTION}

1) Problem. Let $\Omega$ be a bounded polygonal domain in $R^{3}$ with the boundary $\Gamma:=\partial \Omega$ and the unit outward normal $\mathbf{n}$. Let $\epsilon(\mathbf{x})$ and $\mu(\mathbf{x})$ denote the dielectric constant and the magnetic permeability of the material in $\Omega$, respectively. Let $\sigma(\mathbf{x})$ denote the conductivity of the medium. Then, if $\mathbf{E}(\mathbf{x}, t)$ and $\mathbf{H}(\mathbf{x}, t)$ denote, respectively, the electric and magnetic fields, Maxwell's equations [2] state that

$$
\begin{array}{ll}
\epsilon \mathbf{E}_{t}+\sigma \mathbf{E}-\nabla \times \mathbf{H}=-\mathbf{J} & \text { in } \Omega \times(0, T), \\
\mu \mathbf{H}_{t}+\nabla \times \mathbf{E}=0 & \text { in } \Omega \times(0, T),
\end{array}
$$

where $\mathbf{J}=\mathbf{J}(\mathbf{x}, t)$ is a known function specifying the applied current. For simplicity, in this paper we shall assume a perfect conducting boundary condition on $\Omega$ so that

$$
\mathbf{n} \times \mathbf{E}=0 \quad \text { on } \partial \Omega \times(0, T) .
$$

In addition, the initial conditions must be specified so that

$$
\mathbf{E}(\mathbf{x}, 0)=\mathbf{E}_{0}(\mathbf{x}), \quad \mathbf{H}(\mathbf{x}, 0)=\mathbf{H}_{0}(\mathbf{x}), \quad \forall \mathbf{x} \in \Omega
$$

where $\mathbf{E}_{0}$ and $\mathbf{H}_{0}$ are given functions. The coefficients $\epsilon, \mu$ and $\sigma$ are bounded, and there exist constants $\epsilon_{\min }$ and $\mu_{\min }$ such that

$$
0<\epsilon_{\min } \leq \epsilon(\mathbf{x}), \quad 0<\mu_{\min } \leq \mu(\mathbf{x}), \quad \forall \mathbf{x} \in \Omega .
$$

Furthermore, the conductivity $\sigma$ is a nonnegative function on $\bar{\Omega}$.

Received by the editor September 22, 1997 and, in revised form, March 3, 1998.

1991 Mathematics Subject Classification. Primary 65N30; Secondary 35L15.

Key words and phrases. Maxwell's equations, superconvergence, finite element.

(C)1999 American Mathematical Society 
2) Monk's results. P. Monk (see [7] and [8]) described a mixed finite element scheme and a finite element scheme, respectively, for Maxwell's equations, and provided error estimates for smooth solutions as follows:

$$
\begin{gathered}
\left\|\mathbf{H}-\mathbf{H}^{h}\right\|_{0}+\left\|\mathbf{E}-\mathbf{E}^{h}\right\|_{0}=O\left(h^{k}\right), \text { for mixed FEM, } \\
\left\|\nabla \times\left(\mathbf{E}-\mathbf{E}^{h}\right)\right\|_{0}+\left\|\mathbf{E}_{t}-\mathbf{E}_{t}^{h}\right\|_{0}=O\left(h^{k}\right), \text { for FEM, }
\end{gathered}
$$

where $k$ is the order of the finite element space, and $\mathbf{E}^{h}$ and $\mathbf{H}^{h}$ are mixed finite element solutions or finite element solutions of $\mathbf{E}$ and $\mathbf{H}$, respectively.

Moreover, for the equation (1.1) with $\epsilon=\mu=1, \sigma=0$ and the mixed finite element scheme, Monk (see [11]) provided the superconvergence estimate

$$
\mathbf{E}-\mathbf{E}^{h}|\|+\|| \mathbf{H}^{h} \mid \|_{W}=O\left(h^{k+1}\right),
$$

where $\||\cdot|\|$ and $\||\cdot|\|_{W}$ are special mesh dependent discrete norms (see [11] for details).

3) Our results. In this paper, it is shown that when the finite element meshes are structural and the solutions are smooth sufficiently, global superconvergence can be achieved, i.e. the standard error estimates (1.4) and (1.5) can be improved to

$$
\begin{gathered}
\left\|\mathbf{H}-\Pi_{2 h} \mathbf{H}^{h}\right\|_{0}+\left\|\mathbf{E}-\Pi_{2 h} \mathbf{E}^{h}\right\|_{0}=O\left(h^{k+1}\right), \\
\left\|\nabla \times\left(\mathbf{E}-\Pi_{3 h} \mathbf{E}^{h}\right)\right\|_{0}+\left\|\left(\mathbf{E}-\Pi_{2 h} \mathbf{E}^{h}\right)_{t}\right\|_{0}=O\left(h^{k+1}\right),
\end{gathered}
$$

where $\Pi_{2 h}$ and $\Pi_{3 h}$ are the interpolation postprocess operators, which will be described in Section 5.

4) The relationship between above results. Comparing (1.6) and (1.7), it is easy to see that our superconvergence result (1.7) is very close to Monk's result (1.6), but differs in some respects:

a) Because we used the technique of accurate integral identities, the proof of the superconvergence is more direct and easier. In particular, the results of superconvergence can be extended to the problem with variable coefficients $\epsilon, \mu, \sigma$ and a general domain with almost cubic meshes (see Section 6 for details).

b) Our error estimates show that the finite element solution is superconvergent to the interpolant in $L^{2}$ norm, whereas in [11] superconvergence is proved in a special mesh dependent norm.

c) By means of the interpolation postprocessing technique, global superconvergence is provided on the whole domain, not on special points, lines or faces as usual (see $[11],[3], \ldots$ ).

We would like to point out that the technique of the accurate integral identities used in this paper has been used to achieve global superconvergence for standard finite element methods, mixed finite element methods, nonconforming finite element methods, for differential equations, integral-differential equations, integral equations, for elliptic problems, parabolic problems, hyperbolic problems, Stokes problems, $\cdots$ (cf. [5], [6], ... ). It has been shown that this technique (accurate integral identities) and the symmetric technique of A.H. Schatz, I.H. Sloan, and L.B. Wahlbin (see [13], [14]) are powerful tools for achieving superconvergence. We would like to mention here that the accurate integral identities technique combined with the symmetric technique gives an improved superconvergence estimate. 
Throughout the paper, we shall assume that $\mathbf{E}$ and $\mathbf{H}$ are sufficiently smooth; the requirement for the smoothness will be shown by the norms in the cases.

The plan of the paper is as follows. In Section 2, we shall describe the notations to be used in this paper, and the mixed finite element scheme, the finite element scheme for Maxwell's equations and their error estimates shown in [7] and [8]. In Section 3, two lemmas about integral identities with high accuracy will be shown, which are the basis of our paper. Based on the lemmas in Section 3, the superclose analysis is achieved in Section 4. In Section 5, global superconvergence is derived from superclose estimates by using the interpolation postprocess. In Section 6, the results in Sections $3-5$ are extended to problems with variable coefficients and general domains.

\section{Preliminary notations AND Discrete SChemes}

Let us start by defining some notations. We denote the standard Sobolev space by

$$
W^{m, p}(\Omega)=\left\{v \in L^{p}(\Omega) ; \partial^{\alpha} v \in L^{p}(\Omega), \forall|\alpha| \leq m\right\},
$$

equipped with the standard norm

$$
\|v\|_{m, p}=\left(\sum_{\alpha \mid \leq m} \int_{\Omega}\left|\partial^{\alpha} v\right|^{p}\right)^{\frac{1}{p}} .
$$

When $p=2$, we drop the subscript $p$ from the norm and denote the space $W^{m, 2}(\Omega)$ by $H^{m}(\Omega)$, and

$$
H_{0}^{1}(\Omega)=\left\{v \in H^{1}(\Omega) ; v=0 \text { on } \Gamma\right\} .
$$

In addition, let $(\cdot, \cdot)$ denote the $\left[L^{2}(\Omega)\right]^{3}$ inner product.

In our analysis for Maxwell's equations, there are two important spaces of functions:

$$
\begin{aligned}
& H(\operatorname{curl} ; \Omega)=\left\{\mathbf{v} \in\left[L^{2}(\Omega)\right]^{3} ; \nabla \times \mathbf{v} \in\left[L^{2}(\Omega)\right]^{3}\right\}, \\
& H_{0}(\operatorname{curl} ; \Omega)=\{\mathbf{v} \in H(\operatorname{curl} ; \Omega) ; \mathbf{n} \times \mathbf{v}=0 \text { on } \Gamma\},
\end{aligned}
$$

equipped with the norm

$$
\|\mathbf{v}\|_{H^{c}}=\left(\|\mathbf{v}\|_{0}^{2}+\|\nabla \times \mathbf{v}\|_{0}^{2}\right)^{\frac{1}{2}} .
$$

To approximate (1.1)-(1.3), we use finite element spaces $U^{h} \subset\left[L^{2}(\Omega)\right]^{3}$ and $V^{h} \subset H(\operatorname{curl} ; \Omega)$. In addition, $V_{0}^{h} \subset H_{0}(\operatorname{curl} ; \Omega)$. Then, two discrete schemes presented in [7] and [8] are described as follows.

1) A mixed finite element scheme. Multiply equation (1.1) by test functions $\Phi \in\left[L^{2}(\Omega)\right]^{3}$ and $\Psi \in H(\operatorname{curl} ; \Omega)$ and integrate over $\Omega$. Integrating the curl term in the second equation of (1.1) by parts (also using (1.2)), we can obtain the weak form for (1.1)-(1.3) as follows:

$$
\begin{array}{ll}
\left(\epsilon \mathbf{E}_{t}, \Phi\right)+(\sigma \mathbf{E}, \Phi)-(\nabla \times \mathbf{H}, \Phi)=-(\mathbf{J}, \Phi), & \forall \Phi \in\left[L^{2}(\Omega)\right]^{3}, \\
\left(\mu \mathbf{H}_{t}, \Psi\right)+(\mathbf{E}, \nabla \times \Psi)=0, & \forall \Psi \in H(\operatorname{curl} ; \Omega), \\
\mathbf{E}(\mathbf{x}, 0)=\mathbf{E}_{0}(\mathbf{x}), \quad \mathbf{H}(\mathbf{x}, 0)=\mathbf{H}_{0}(\mathbf{x}) . &
\end{array}
$$


Then, a mixed finite element scheme is to find $\mathbf{E}^{h} \in U^{h}, \mathbf{H}^{h} \in V^{h}$ such that

$$
\begin{array}{ll}
\left(\epsilon \mathbf{E}_{t}^{h}, \Phi\right)+\left(\sigma \mathbf{E}^{h}, \Phi\right)-\left(\nabla \times \mathbf{H}^{h}, \Phi\right)=-(\mathbf{J}, \Phi), & \forall \Phi \in U^{h}, \\
\left(\mu \mathbf{H}_{t}^{h}, \Psi\right)+\left(\mathbf{E}^{h}, \nabla \times \Psi\right)=0, & \forall \Psi \in V^{h}, \\
\mathbf{E}^{h}(0)=\mathbf{E}_{0}^{I}, \quad \mathbf{H}^{h}(0)=\mathbf{H}_{0}^{I} . &
\end{array}
$$

where $\mathbf{E}_{0}^{I} \in U^{h}$ and $\mathbf{H}_{0}^{I} \in V^{h}$ are interpolations of $\mathbf{E}_{0}$ and $\mathbf{H}_{0}$, respectively.

The existence and uniqueness for the above equations have been shown in [7] with the error estimate

$$
\begin{aligned}
& \left\|\left(\mathbf{H}-\mathbf{H}^{h}\right)(t)\right\|_{0}+\left\|\left(\mathbf{E}-\mathbf{E}^{h}\right)(t)\right\|_{0} \\
& \quad \leq C h^{k}\left[\|\mathbf{H}(0)\|_{k+1}+\|\mathbf{E}(0)\|_{k}+\left(\int_{0}^{t}\left(\left\|\mathbf{H}_{t}\right\|_{k}^{2}+\|\mathbf{H}\|_{k+1}^{2}+\left\|\mathbf{E}_{t}\right\|_{k}^{2}+\|\mathbf{E}\|_{k}^{2}\right) d t\right)^{\frac{1}{2}}\right],
\end{aligned}
$$

where $k$ is the order of the mixed finite element space.

2) A finite element scheme. Another approach to approximating (1.1) is to derive a second-order hyperbolic problem for $\mathbf{E}(\mathbf{x}, t)$. By taking the time derivate of the first equation and using the second equation in (1.1), we obtain the following electric field equation:

$$
\epsilon \mathbf{E}_{t t}+\sigma \mathbf{E}_{t}+\nabla \times\left(\frac{1}{\mu} \nabla \times \mathbf{E}\right)=\mathbf{G} \quad \text { in } \Omega \times(0, T),
$$

where $\mathbf{G}(\mathbf{x}, t)=-\mathbf{J}_{t}(\mathbf{x}, t)$. Also using (1.1) and (1.3) we obtain the initial condition

$$
\mathbf{E}_{t}(\mathbf{x}, 0)=\mathbf{E}_{t 0}(\mathbf{x}) \equiv \frac{1}{\epsilon(\mathbf{x})}\left[\mathbf{J}(\mathbf{x}, 0)+\nabla \times \mathbf{H}_{0}(\mathbf{x})-\sigma(\mathbf{x}) \mathbf{E}_{0}(\mathbf{x})\right] .
$$

Multiplying (2.4) by a test function $\Phi \in \mathbf{H}_{0}(\operatorname{curl} ; \Omega)$ and integrating the curl term by parts, we obtain the weak form for (2.4) as follows:

$$
\left(\epsilon \mathbf{E}_{t t}, \Phi\right)+\left(\sigma \mathbf{E}_{t}, \Phi\right)+\left(\frac{1}{\mu} \nabla \times \mathbf{E}, \nabla \times \Phi\right)=(\mathbf{G}, \Phi), \quad \forall \Phi \in \mathbf{H}_{0}(\operatorname{curl} ; \Omega),
$$

subject to the initial conditions

$$
\mathbf{E}(0)=\mathbf{E}_{0} \quad \text { and } \quad \mathbf{E}_{t}(0)=\mathbf{E}_{t 0} .
$$

The finite element scheme is to find $\mathbf{E}^{h} \in V_{0}^{h}$, such that

$$
\left(\epsilon \mathbf{E}_{t t}^{h}, \Phi\right)+\left(\sigma \mathbf{E}_{t}^{h}, \Phi\right)+\left(\frac{1}{\mu} \nabla \times \mathbf{E}^{h}, \nabla \times \Phi\right)=(\mathbf{G}, \Phi), \quad \forall \Phi \in V_{0}^{h},
$$

subject to the initial conditions

$$
\mathbf{E}^{h}(0)=\mathbf{E}_{0}^{I} \quad \text { and } \quad \mathbf{E}_{t}^{h}(0)=\mathbf{E}_{t 0}^{I},
$$

where $\mathbf{E}_{0}^{I}$ and $\mathbf{E}_{t 0}^{I}$ are interpolations of $\mathbf{E}_{0}$ and $\mathbf{E}_{t 0}$, respectively.

The existence and uniqueness for (2.6) and (2.8) have been shown in [8] with the error estimate

$$
\begin{aligned}
\left\|\left(\mathbf{E}-\mathbf{E}^{h}\right)(t)\right\|_{H^{c}}+\left\|\left(\mathbf{E}-\mathbf{E}^{h}\right)_{t}(t)\right\|_{0} \\
\leq C\left(\left\|\left(\mathbf{E}-\mathbf{E}^{h}\right)(0)\right\|_{H^{c}}+\left\|\left(\mathbf{E}-\mathbf{E}^{h}\right)_{t}(0)\right\|_{0}\right. \\
\left.\quad+h^{k}\left(\max _{0 \leq s \leq t}\left\|\mathbf{E}_{t}(s)\right\|_{k+1}+\int_{0}^{t}\left\|\mathbf{E}_{t t}\right\|_{k+1} d s\right)\right),
\end{aligned}
$$

where $k$ is the order of the finite element spaces. 


\section{TWO FUnDAMENTAL LEMMAS}

In order to concentrate our attention on the primary idea, in this and the next section we shall assume that $\Omega$ is a cubic domain. Let $T_{h}$ be a cubic mesh on $\Omega$ with the largest size $h$, and let the mixed finite element space ([12]) be

$$
\begin{aligned}
U^{h} & =\left\{\Phi \in\left[L^{2}(\Omega)\right]^{3} ;\left.\Phi\right|_{e} \in Q_{k, k-1, k-1} \times Q_{k-1, k, k-1} \times Q_{k-1, k-1, k}, \forall e \in T_{h}\right\}, \\
V^{h} & =\left\{\Psi \in H(\operatorname{curl} ; \Omega) ;\left.\Psi\right|_{e} \in Q_{k-1, k, k} \times Q_{k, k-1, k} \times Q_{k, k, k-1}, \forall e \in T_{h}\right\}
\end{aligned}
$$

where $Q_{i, j, k}$ is a space of polynomials whose degrees for $x, y, z$ are less than or equal to $i, j, k$, respectively.

In addition, define $\mathbf{E}^{I} \in U^{h}$ and $\mathbf{H}^{I} \in V^{h}$ to be the interpolations of $\mathbf{E}$ and $\mathbf{H}$ satisfying

$$
\int_{e}\left(\mathbf{E}-\mathbf{E}^{I}\right) \cdot \Phi=0, \quad \forall \Phi \in U^{h}
$$

and

$$
\begin{aligned}
& \int_{l_{i}}\left(\mathbf{H}-\mathbf{H}^{I}\right) \cdot \mathbf{t} q d l=0, \quad \forall q \in P_{k}, \quad i=1, \cdots, 12, \\
& \int_{\sigma_{i}}\left(\left(\mathbf{H}-\mathbf{H}^{I}\right) \times \mathbf{n}\right) \cdot \mathbf{q} d \sigma=0, \quad \forall \mathbf{q} \in Q_{k-2, k-1} \times Q_{k-1, k-2}, \quad i=1, \cdots, 6, \\
& \int_{e}\left(\mathbf{H}-\mathbf{H}^{I}\right) \cdot \mathbf{q}=0, \quad \forall \mathbf{q} \in Q_{k-1, k-2, k-2} \times Q_{k-2, k-1, k-2} \times Q_{k-2, k-2, k-1},
\end{aligned}
$$

where $l_{i}, \sigma_{i}$ are edges and surfaces of the element $e, \mathbf{t}, \mathbf{n}$ are tangent vector and normal vector, respectively, and $P_{k}$ is a polynomial function space of order $k$.

On the cubic element $e=\left[x_{e}-h_{e}, x_{e}+h_{e}\right] \times\left[y_{e}-k_{e}, y_{e}+k_{e}\right] \times\left[z_{e}-d_{e}, z_{e}+d_{e}\right]$, define

$$
A(x)=\frac{\left(x-x_{e}\right)^{2}-h_{e}^{2}}{2}, \quad B(y)=\frac{\left(y-y_{e}\right)^{2}-k_{e}^{2}}{2}, \quad D(z)=\frac{\left(z-z_{e}\right)^{2}-d_{e}^{2}}{2} .
$$

Then, we can obtain some integral identities with high accuracy by means of the functions $A(x), B(y), D(z)$ and the interpolation conditions.

\section{Lemma 3.1.}

$$
\int_{\Omega} \nabla \times\left(\mathbf{H}-\mathbf{H}^{I}\right) \cdot \Phi=O\left(h^{k+1}\right)\|\mathbf{H}\|_{k+2}\|\Phi\|_{0}, \quad \forall \Phi \in U^{h} .
$$

Proof. Let $\mathbf{w}=\mathbf{H}-\mathbf{H}^{I}$, and $\mathbf{w}=\left(w_{1}, w_{2}, w_{3}\right), \Phi=\left(\phi_{1}, \phi_{2}, \phi_{3}\right) ;$ then

$$
(\nabla \times \mathbf{w})_{1} \phi_{1}=\left(\partial_{y} w_{3}-\partial_{z} w_{2}\right) \phi_{1}
$$

where $\partial_{y}=\frac{\partial}{\partial y}, \partial_{z}=\frac{\partial}{\partial z}, \phi_{1} \in Q_{k, k-1, k-1}$.

When $k=1$ and $\phi_{1} \in Q_{1,0,0}$ we have

$$
\phi_{1}=\phi_{1}\left(x_{e}, y, z\right)+\left(x-x_{e}\right) \partial_{x} \phi_{1}
$$




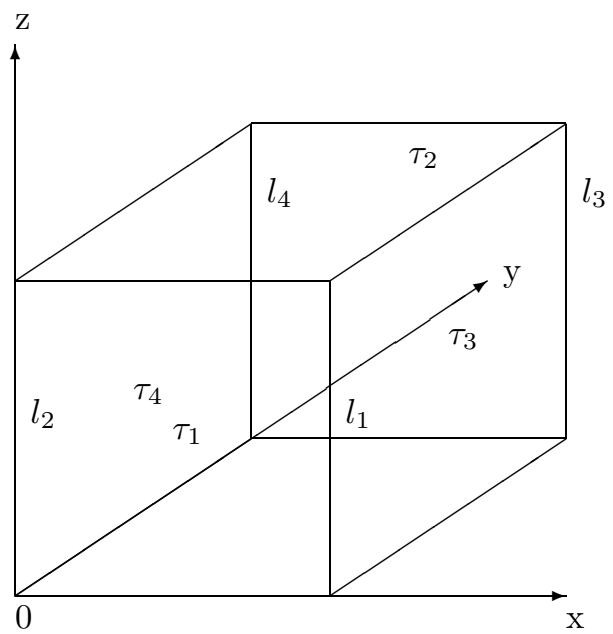

FIGURE 1

By the interpolation conditions of $\mathbf{H}^{I}$ and noting that $A(x)=0$ when $x=x_{e} \pm h_{e}$,

$$
\begin{aligned}
\int_{e} \partial_{y} w_{3}=\left(\int_{\tau_{2}}-\int_{\tau_{1}}\right) w_{3} d x d z=\left(\int_{\tau_{2}}-\int_{\tau_{1}}\right) A^{\prime \prime}(x) w_{3} d x d z \\
=\left(\int_{l_{3}}-\int_{l_{4}}-\int_{l_{1}}+\int_{l_{2}}\right) A^{\prime}(x) w_{3} d z-\left(\int_{\tau_{2}}-\int_{\tau_{1}}\right) A^{\prime}(x) \partial_{x} w_{3} d x d z \\
=0-\left(\int_{l_{3}}-\int_{l_{4}}-\int_{l_{1}}+\int_{l_{2}}\right) A(x) \partial_{x} w_{3} d z+\left(\int_{\tau_{2}}-\int_{\tau_{1}}\right) A(x) \partial_{x}^{2} w_{3} d x d z \\
=0+\int_{e} A(x) \partial_{x}^{2} \partial_{y} H_{3},
\end{aligned}
$$

where $\tau_{1}, \tau_{2}, l_{1}, \cdots, l_{4}$ are surfaces and edges of the element $e$ (see Figure 1 ).

In a similar way, it can be proved that

$$
\int_{e} \partial_{y} w_{3}\left(x-x_{e}\right)=\frac{1}{6} \int_{e}\left(A^{2}(x)\right)^{\prime \prime \prime} \partial_{y} w_{3}=\frac{1}{6} \int_{e}\left(A^{2}(x)\right)^{\prime} \partial_{x}^{2} \partial_{y} H_{3}
$$

Note that

$$
\phi_{1}\left(x_{e}, y, z\right)=\phi_{1}-\left(x-x_{e}\right) \partial_{x} \phi_{1} .
$$

By (3.3)-(3.5) and the inverse inequality [1], it can be proved that

$$
\begin{aligned}
\int_{e} \partial_{y} w_{3} \phi_{1} & =\int_{e}\left(A(x) \phi_{1}\left(x_{e}, y, z\right)+\frac{1}{6}\left(A^{2}(x)\right)^{\prime} \partial_{x} \phi_{1}\right) \partial_{x}^{2} \partial_{y} w_{3} \\
& =\int_{e} A(x)\left(\phi_{1}-\frac{2}{3}\left(x-x_{e}\right) \partial_{x} \phi_{1}\right) \partial_{x}^{2} \partial_{y} H_{3} \\
& =O\left(h^{2}\right)\left\|H_{3}\right\|_{3, e}\left\|\phi_{1}\right\|_{0, e} .
\end{aligned}
$$

Similarly, we can obtain the same results for $\int_{e} \partial_{z} w_{2} \phi_{1}$ and $\int_{e}(\nabla \times \mathbf{w})_{i} \phi_{i}, i=2,3$. Hence, (3.1) is proved for $k=1$. 
When $k \geq 2$,

$$
\begin{aligned}
\phi_{1}= & \sum_{i=1}^{k-2} \frac{\left(x-x_{e}\right)^{i}}{i !} \partial_{x}^{i} \phi_{1}\left(x_{e}, y, z\right)+\frac{\left(x-x_{e}\right)^{k-1}}{(k-1) !} \partial_{x}^{k-1} \phi_{1}\left(x_{e}, y, z\right) \\
& +\frac{\left(x-x_{e}\right)^{k}}{k !} \partial_{x}^{k} \phi_{1} .
\end{aligned}
$$

By the interpolation conditions for $\mathbf{H}^{I}$,

$$
\begin{aligned}
\int_{e} \partial_{y} w_{3} & \sum_{i=0}^{k-2} \frac{\left(x-x_{e}\right)^{i}}{i !} \partial_{x}^{i} \phi_{1}\left(x_{e}, y, z\right) \\
= & \left(\int_{\tau_{2}}-\int_{\tau_{1}}\right) w_{3} \sum_{i=0}^{k-2} \frac{\left(x-x_{e}\right)^{i}}{i !} \partial_{x}^{i} \phi_{1}\left(x_{e}, y, z\right) d x d z \\
& -\int_{e} w_{3} \sum_{i=0}^{k-2} \frac{\left(x-x_{e}\right)^{i}}{i !} \partial_{x}^{i} \partial_{y} \phi_{1}\left(x_{e}, y, z\right)=0 .
\end{aligned}
$$

Note that

$$
\frac{\left(x-x_{e}\right)^{k-1}}{(k-1) !}=\frac{2^{k}}{(2 k) !}\left(A^{k}(x)\right)^{(k+1)}+F(x)
$$

where $\left(A^{k}(x)\right)^{(k+1)}$ is a derivative of order $k+1$ for $A^{k}(x), F(x) \in P_{k-3}$. We can obtain that

$$
\begin{aligned}
\int_{e} \partial_{y} w_{3} & \frac{\left(x-x_{e}\right)^{k-1}}{(k-1) !} \partial_{x}^{k-1} \phi_{1}\left(x_{e}, y, z\right) \\
= & \frac{2^{k}}{(2 k) !} \int_{e}\left(A^{k}(x)\right)^{(k+1)} \partial_{y} w_{3} \partial_{x}^{k-1} \phi_{1}\left(x_{e}, y, z\right) \\
& +\int_{e} F(x) \partial_{y} w_{3} \partial_{x}^{k-1} \phi_{1}\left(x_{e}, y, z\right) \\
= & \frac{2^{k}}{(2 k) !}\left(\int_{\tau_{3}}-\int_{\tau_{4}}\right)\left(A^{k}(x)\right)^{(k)} \partial_{y} w_{3} \partial_{x}^{k-1} \phi_{1}\left(x_{e}, y, z\right) d y d z \\
& --\frac{2^{k}}{(2 k) !} \int_{e}\left(A^{k}(x)\right)^{(k)} \partial_{x} \partial_{y} w_{3} \partial_{x}^{k-1} \phi_{1}\left(x_{e}, y, z\right)+0 \\
= & \frac{2^{k}}{(2 k) !}\left(\int_{l_{3}}-\int_{l_{1}}-\int_{l_{4}}+\int_{l_{2}}\right)\left(A^{k}(x)\right)^{(k)} w_{3} \partial_{x}^{k-1} \phi_{1}\left(x_{e}, y, z\right) d z \\
& -\frac{2^{k}}{(2 k) !}\left(\int_{\tau_{3}}-\int_{\tau_{4}}\right)\left(A^{k}(x)\right)^{(k)} w_{3} \partial_{x}^{k-1} \partial_{y} \phi_{1}\left(x_{e}, y, z\right) d y d z \\
& -\frac{(-2)^{k}}{(2 k) !} \int_{e} A^{k}(x) \partial_{x}^{k+1} \partial_{y} H_{3} \partial_{x}^{k-1} \phi_{1}\left(x_{e}, y, z\right) \\
= & 0+0+O\left(h^{2 k}\right)\left\|H_{3}\right\|_{k+2, e}\left\|\phi_{1}\right\|_{k-1, e} \\
= & O\left(h^{k+1}\right)\left\|H_{3}\right\|_{k+2, e}\left\|\phi_{1}\right\|_{0, e} .
\end{aligned}
$$


In the same way,

$$
\begin{aligned}
\int_{e} \partial_{y} w_{3} \frac{\left(x-x_{e}\right)^{k}}{k !} \partial_{x}^{k} \phi_{1} & =\frac{(-2)^{k+1}}{(2 k+2) !} \int_{e}\left(A^{k+1}(x)\right)^{\prime} \partial_{x}^{k+1} \partial_{y} H_{3} \partial_{x}^{k} \phi_{1} \\
& =O\left(h^{k+1}\right)\left\|H_{3}\right\|_{k+2, e}\left\|\phi_{1}\right\|_{0, e} .
\end{aligned}
$$

So, by (3.6)-(3.10),

$$
\int_{\Omega} \partial_{y} w_{3} \phi_{1}=O\left(h^{k+1}\right)\left\|H_{3}\right\|_{k+1}\left\|\phi_{1}\right\|_{0}
$$

In the same way, we can prove the samilar results for the terms $\int_{e} \partial_{z} w_{2} \phi_{1}$ and $\int_{e}(\nabla \times \mathbf{w})_{i} \phi_{i}, i=2,3$, for $k \geq 2$. Hence, the proof of Lemma 3.1 is completed.

\section{Lemma 3.2.}

$$
\int_{\Omega}\left(\mathbf{H}-\mathbf{H}^{I}\right) \Psi=O\left(h^{k+1}\right)\|\mathbf{H}\|_{k+1}\|\Psi\|_{0}, \quad \forall \Psi \in V^{h} .
$$

Proof. Let $\mathbf{w}=\mathbf{H}-\mathbf{H}^{I}$. When $k=1$ and $\psi_{3} \in Q_{1,1,0}$, we have

$$
\begin{aligned}
\psi_{3}= & \psi_{3}\left(x_{e}, y_{e}, z\right)+\left(x-x_{e}\right) \partial_{x} \psi_{3}\left(x, y_{e}, z\right)+\left(y-y_{e}\right) \partial_{y} \psi_{3}\left(x_{e}, y, z\right) \\
& +\left(x-x_{e}\right)\left(y-y_{e}\right) \partial_{x} \partial_{y} \psi_{3} .
\end{aligned}
$$

In the similar way as for Lemma 3.1, it is easy to show that

$$
\begin{gathered}
\int_{e} w_{3}=-\int_{e} A^{\prime}(x) B^{\prime}(y) \partial_{x} \partial_{y} w_{3}+\int_{e} A(x) \partial_{x}^{2} H_{3}+\int_{e} B(y) \partial_{y}^{2} H_{3}, \\
\int_{e}\left(x-x_{e}\right) w_{3}=-\frac{h_{e}^{2}}{3} \int_{e} B^{\prime}(y) \partial_{x} \partial_{y} w_{3}+\frac{1}{6} \int_{e}\left(A^{2}(x)\right)^{\prime} \partial_{x}^{2} H_{3}, \\
\int_{e}\left(y-y_{e}\right) w_{3}=-\frac{k_{e}^{2}}{3} \int_{e} A^{\prime}(x) \partial_{x} \partial_{y} w_{3}+\frac{1}{6} \int_{e}\left(B^{2}(y)\right)^{\prime} \partial_{y}^{2} H_{3}, \\
\int_{e}\left(x-x_{e}\right)\left(y-y_{e}\right) w_{3}=\int_{e} A(x) B(y) \partial_{x} \partial_{y} w_{3} .
\end{gathered}
$$


By (3.12)-(3.16), and note that $\left\|\partial_{x} \partial_{y} w_{3}\right\|_{0} \leq C\left\|H_{3}\right\|_{2}$,

$$
\int_{e} w_{3} \psi_{3}=O\left(h^{2}\right)\left\|H_{3}\right\|_{2, e}\left\|\psi_{3}\right\|_{0, e}
$$

Similar results can be proved for $\int_{e} w_{i} \psi_{i}, i=1,2$. Hence, (3.11) follows for $k=1$.

When $k \geq 2$,

$$
\begin{aligned}
\psi_{3}= & \sum_{i=0}^{k-2} \sum_{j=0}^{k-2} \frac{\left(x-x_{e}\right)^{i}}{i !} \frac{\left(y-y_{e}\right)^{j}}{j !} \partial_{x}^{i} \partial_{y}^{j} \psi_{3}\left(x_{e}, y_{e}, z\right) \\
& +\frac{\left(x-x_{e}\right)^{k-1}}{(k-1) !} \sum_{j=0}^{k-2} \frac{\left(y-y_{e}\right)^{j}}{j !} \partial_{x}^{k-1} \partial_{y}^{j} \psi_{3}\left(x_{e}, y_{e}, z\right) \\
& +\frac{\left(x-x_{e}\right)^{k}}{k !} \sum_{j=0}^{k-2} \frac{\left(y-y_{e}\right)^{j}}{j !} \partial_{x}^{k} \partial_{y}^{j} \psi_{3}\left(x_{e}, y_{e}, z\right) \\
& +\frac{\left(y-y_{e}\right)^{k-1}}{(k-1) !} \sum_{i=0}^{k-2} \frac{\left(x-x_{e}\right)^{i}}{i !} \partial_{x}^{i} \partial_{y}^{k-1} \psi_{3}\left(x_{e}, y_{e}, z\right) \\
& +\frac{\left(y-y_{e}\right)^{k}}{k !} \sum_{i=0}^{k-2} \frac{\left(x-x_{e}\right)^{i}}{i !} \partial_{x}^{i} \partial_{y}^{k} \psi_{3}\left(x_{e}, y_{e}, z\right) \\
& +\frac{\left(x-x_{e}\right)^{k-1}}{(k-1) !} \frac{\left(y-y_{e}\right)^{k-1}}{(k-1) !} \partial_{x}^{k-1} \partial_{y}^{k-1} \psi_{3}\left(x_{e}, y_{e}, z\right) \\
& +\frac{\left(x-x_{e}\right)^{k}}{k !} \frac{\left(y-y_{e}\right)^{k-1}}{(k-1) !} \partial_{x}^{k} \partial_{y}^{k-1} \psi_{3}\left(x_{e}, y_{e}, z\right) \\
& +\frac{\left(x-x_{e}\right)^{k-1}}{(k-1) !} \frac{\left(y-y_{e}\right)^{k}}{k !} \partial_{x}^{k-1} \partial_{y}^{k} \psi_{3}\left(x_{e}, y_{e}, z\right) \\
+ & \frac{\left(x-x_{e}\right)^{k}}{k !} \frac{\left(y-y_{e}\right)^{k}}{k !} \partial_{x}^{k} \partial_{y}^{k} \psi_{3}\left(x_{e}, y_{e}, z\right) .
\end{aligned}
$$

By the definition of interpolation for $\mathbf{H}^{I}$,

$$
\left(w_{3}, \sum_{i=0}^{k-2} \sum_{j=0}^{k-2} \frac{\left(x-x_{e}\right)^{i}}{i !} \frac{\left(y-y_{e}\right)^{j}}{j !} \partial_{x}^{i} \partial_{y}^{j} \psi_{3}\left(x_{e}, y_{e}, z\right)\right)=0
$$


From (3.8) and (3.18), integrating by parts, it can be proved that

$$
\begin{aligned}
\int_{e} w_{3} & \frac{\left(x-x_{e}\right)^{k-1}}{(k-1) !} \sum_{j=0}^{k-2} \frac{\left(y-y_{e}\right)^{j}}{j !} \partial_{x}^{k-1} \partial_{y}^{j} \psi_{3}\left(x_{e}, y_{e}, z\right) \\
= & \int_{e} w_{3} \frac{2^{k}}{(2 k) !}\left(A^{k}(x)\right)^{(k+1)} \sum_{j=0}^{k-2} \frac{\left(y-y_{e}\right)^{j}}{j !} \partial_{x}^{k-1} \partial_{y}^{j} \psi_{3}\left(x_{e}, y_{e}, z\right)+0 \\
= & \left(\int_{\tau_{3}}-\int_{\tau_{4}}\right) w_{3} \frac{2^{k}}{(2 k) !}\left(A^{k}(x)\right)^{(k)} \sum_{j=0}^{k-2} \frac{\left(y-y_{e}\right)^{j}}{j !} \partial_{x}^{k-1} \partial_{y}^{j} \psi_{3}\left(x_{e}, y_{e}, z\right) d y d z \\
& -\int_{e} \partial_{x} w_{3} \frac{2^{k}}{(2 k) !}\left(A^{k}(x)\right)^{(k)} \sum_{j=0}^{k-2} \frac{\left(y-y_{e}\right)^{j}}{j !} \partial_{x}^{k-1} \partial_{y}^{j} \psi_{3}\left(x_{e}, y_{e}, z\right) \\
= & 0-\frac{(-2)^{k}}{(2 k) !} \int_{e} \partial_{x}^{k+1} H_{3} A^{k}(x) \sum_{j=0}^{k-2} \frac{\left(y-y_{e}\right)^{j}}{j !} \partial_{x}^{k-1} \partial_{y}^{j} \psi_{3}\left(x_{e}, y_{e}, z\right) \\
= & O\left(h^{2 k}\right)\left\|H_{3}\right\|_{k+1, e}\left\|\psi_{3}\right\|_{k-1, e}=O\left(h^{k+1}\right)\left\|H_{3}\right\|_{k+1, e}\left\|\psi_{3}\right\|_{0, e} .
\end{aligned}
$$

In the same way,

$$
\begin{array}{r}
\int_{e} w_{3} \frac{\left(y-y_{e}\right)^{k-1}}{(k-1) !} \sum_{i=0}^{k-2} \frac{\left(x-x_{e}\right)^{i}}{i !} \partial_{x}^{i} \partial_{y}^{k-1} \psi_{3}\left(x_{e}, y_{e}, z\right) \\
=O\left(h^{k+1}\right)\left\|H_{3}\right\|_{k+1, e}\left\|\psi_{3}\right\|_{0, e}
\end{array}
$$

$$
\begin{gathered}
\int_{e} w_{3} \frac{\left(y-y_{e}\right)^{k}}{k !} \sum_{i=0}^{k-2} \frac{\left(x-x_{e}\right)^{i}}{i !} \partial_{x}^{i} \partial_{y}^{k} \psi_{3}\left(x_{e}, y_{e}, z\right) \\
=O\left(h^{k+1}\right)\left\|H_{3}\right\|_{k+1, e}\left\|\psi_{3}\right\|_{0, e} .
\end{gathered}
$$


For the sixth term in the right of (3.17), when $k=2$,

$$
\begin{aligned}
\int_{e} w_{3} & \frac{\left(x-x_{e}\right)^{k-1}}{(k-1) !} \frac{\left(y-y_{e}\right)^{k-1}}{(k-1) !} \partial_{x}^{k-1} \partial_{y}^{k-1} \psi_{3}\left(x_{e}, y_{e}, z\right) \\
= & \int_{e} w_{3}\left(x-x_{e}\right)\left(y-y_{e}\right) \partial_{x} \partial_{y} \psi_{3}\left(x_{e}, y_{e}, z\right) \\
= & \frac{1}{6} \int_{e}\left(A^{2}(x)\right)^{\prime \prime \prime}\left(y-y_{e}\right) w_{3} \partial_{x} \partial_{y} \psi_{3}\left(x_{e}, y_{e}, z\right) \\
= & -\frac{1}{6} \int_{e}\left(A^{2}(x)\right)^{\prime \prime}\left(y-y_{e}\right) \partial_{x} w_{3} \partial_{x} \partial_{y} \psi_{3}\left(x_{e}, y_{e}, z\right) \\
& +\frac{1}{6}\left(\int_{\tau_{3}}-\int_{\tau_{4}}\right)\left(A^{2}(x)\right)^{\prime \prime} \frac{1}{6}\left(B^{2}(y)\right)^{\prime \prime \prime} w_{3} \partial_{x} \partial_{y} \psi_{3}\left(x_{e}, y_{e}, z\right) d y d z \\
= & -\frac{1}{6} \int_{e} A^{2}(x)\left(y-y_{e}\right) \partial_{x}^{3} w_{3} \partial_{x} \partial_{y} \psi_{3}\left(x_{e}, y_{e}, z\right) \\
& -\frac{1}{6} \frac{h_{e}^{2}}{3}\left(\int_{\tau_{3}}-\int_{\tau_{4}}\right)\left(B^{2}(y)\right)^{\prime \prime} \partial_{y} w_{3} \partial_{x} \partial_{y} \psi_{3}\left(x_{e}, y_{e}, z\right) d y d z \\
& +\frac{1}{6} \frac{h_{e}^{2}}{3}\left(\int_{l_{3}}-\int_{l_{1}}-\int_{l_{4}}+\int_{l_{2}}\right)\left(B^{2}(y)\right)^{\prime \prime} w_{3} \partial_{x} \partial_{y} \psi_{3}\left(x_{e}, y_{e}, z\right) d z \\
= & -\frac{1}{6} \int_{e} A^{2}(x)\left(y-y_{e}\right) \partial_{x}^{3} H_{3} \partial_{x} \partial_{y} \psi_{3}\left(x_{e}, y_{e}, z\right) \\
& +\frac{h_{e}^{2}}{18} \int_{e}\left(B^{2}(y)\right)^{\prime} \partial_{x} \partial_{y}^{2} w_{3} \partial_{x} \partial_{y} \psi_{3}\left(x_{e}, y_{e}, z\right)+0 \\
= & O\left(h^{5}\right)\left\|H_{3}\right\|_{3, e}\left\|\psi_{3}\right\|_{2, e}=O\left(h^{3}\right)\left\|H_{3}\right\|_{3, e}\left\|\psi_{3}\right\|_{0, e} .
\end{aligned}
$$

Note that

$$
\frac{\left(x-x_{e}\right)^{k-1}}{(k-1) !}=\frac{2^{k-1}}{(2 k-2) !}\left(A^{k-1}(x)\right)^{(k-1)}+\tilde{F}(x), \quad \tilde{F}(x) \in P_{k-3} .
$$

Hence, when $k \geq 3, k-3 \geq 0$, by (3.19),

$$
\begin{aligned}
\int_{e} w_{3} & \frac{\left(x-x_{e}\right)^{k-1}}{(k-1) !} \frac{\left(y-y_{e}\right)^{k-1}}{(k-1) !} \partial_{x}^{k-1} \partial_{y}^{k-1} \psi_{3}\left(x_{e}, y_{e}, z\right) \\
= & \frac{2^{2 k-2}}{((2 k-2) !)^{2}} \int_{e}\left(A^{k-1}(x)\right)^{(k-1)}\left(B^{k-1}(y)\right)^{(k-1)} w_{3} \\
& \times \partial_{x}^{k-1} \partial_{y}^{k-1} \psi_{3}\left(x_{e}, y_{e}, z\right)+O\left(h^{k+1}\right)\left\|H_{3}\right\|_{k+1, e}\left\|\psi_{3}\right\|_{0, e} \\
= & \frac{(-1)^{k+1} 2^{2 k-2}}{((2 k-2) !)^{2}} \int_{e} A^{k-1}(x)\left(B^{k-1}(y)\right)^{(k-3)} \partial_{x}^{k-1} \partial_{y}^{2} w_{3} \\
& \times \partial_{x}^{k-1} \partial_{y}^{k-1} \psi_{3}\left(x_{e}, y_{e}, z\right)+O\left(h^{k+1}\right)\left\|H_{3}\right\|_{k+1, e}\left\|\psi_{3}\right\|_{0, e} \\
= & O\left(h^{3 k-1}\right)\left\|H_{3}\right\|_{k+1, e}\left\|\psi_{3}\right\|_{2 k-2, e}+O\left(h^{k+1}\right)\left\|H_{3}\right\|_{k+1, e}\left\|\psi_{3}\right\|_{0, e} \\
= & O\left(h^{k+1}\right)\left\|H_{3}\right\|_{k+1, e}\left\|\psi_{3}\right\|_{0, e} .
\end{aligned}
$$


In the same way, when $k \geq 2$,

$$
\begin{aligned}
\int_{e} w_{3} & \frac{\left(x-x_{e}\right)^{k}}{k !} \frac{\left(y-y_{e}\right)^{k-1}}{(k-1) !} \partial_{x}^{k} \partial_{y}^{k-1} \psi_{3}\left(x_{e}, y_{e}, z\right) \\
= & \frac{(-1)^{k+1} 2^{2 k-1}}{(2 k-2) !(2 k) !} \int_{e} A^{k}(x)\left(B^{k-1}(y)\right)^{(k-2)} \partial_{x}^{k} \partial_{y} w_{3} \partial_{x}^{k} \partial_{y}^{k-1} \psi_{3}\left(x_{e}, y_{e}, z\right) \\
& +O\left(h^{k+1}\right)\left\|H_{3}\right\|_{k+1, e}\left\|\psi_{3}\right\|_{0, e} \\
= & O\left(h^{k+1}\right)\left\|H_{3}\right\|_{k+1, e}\left\|\psi_{3}\right\|_{0, e} .
\end{aligned}
$$

Similarly,

$$
\int_{e} w_{3} \frac{\left(x-x_{e}\right)^{k-1}}{(k-1) !} \frac{\left(y-y_{e}\right)^{k}}{k !} \partial_{x}^{k-1} \partial_{y}^{k} \psi_{3}\left(x_{e}, y_{e}, z\right)=O\left(h^{k+1}\right)\left\|H_{3}\right\|_{k+1, e}\left\|\psi_{3}\right\|_{0, e},
$$

$$
\int_{e} w_{3} \frac{\left(x-x_{e}\right)^{k}}{k !} \frac{\left(y-y_{e}\right)^{k}}{k !} \partial_{x}^{k} \partial_{y}^{k} \psi_{3}\left(x_{e}, y_{e}, z\right)=O\left(h^{k+1}\right)\left\|H_{3}\right\|_{k+1, e}\left\|\psi_{3}\right\|_{0, e} .
$$

So, by (3.17)-(3.27),

$$
\int_{\Omega} w_{3} \psi_{3}=O\left(h^{k+1}\right)\left\|H_{3}\right\|_{k+1}\left\|\psi_{3}\right\|_{0} .
$$

Similar results can be proved for $\int_{e} w_{i} \psi_{i}, i=1,2$, when $k \geq 2$. Hence (3.11) follows.

\section{Superclose estimates}

Now, based on the lemmas in Section 3, we can get the principal results in this paper. In this section, we will assume that $\epsilon=\mu=1$ and $\sigma=0$ for equation (1.1).

Theorem 4.1. Let $\boldsymbol{E}, \boldsymbol{H}, \boldsymbol{E}^{h}, \boldsymbol{H}^{h}$ be the solutions of (2.1) and (2.2), respectively, and $\boldsymbol{E}^{I} \in U^{h}, \boldsymbol{H}^{I} \in V^{h}$ be the interpolations of $\boldsymbol{E}$ and $\boldsymbol{H}$. Then

$$
\left\|\mathbf{E}^{h}-\mathbf{E}^{I}\right\|_{0}+\left\|\mathbf{H}^{h}-\mathbf{H}^{I}\right\|_{0}=O\left(h^{k+1}\right)\left(\int_{0}^{t}\left(\|\mathbf{H}\|_{k+2}^{2}+\left\|\mathbf{H}_{t}\right\|_{k+1}^{2}\right) d s\right)^{\frac{1}{2}} .
$$

Proof. Let $\xi=\mathbf{E}^{h}-\mathbf{E}^{I}, \eta=\mathbf{H}^{h}-\mathbf{H}^{I}$; then by (2.1) and (2.2),

$$
\begin{aligned}
\frac{1}{2} \frac{d}{d t}( & \left.\|\xi\|_{0}^{2}+\|\eta\|_{0}^{2}\right)=\left(\xi_{t}, \xi\right)+\left(\eta_{t}, \eta\right) \\
= & \left(\xi_{t}, \xi\right)-(\nabla \times \eta, \xi)+\left(\eta_{t}, \eta\right)+(\xi, \nabla \times \eta) \\
= & \left(\left(\mathbf{E}-\mathbf{E}^{I}\right)_{t}, \xi\right)-\left(\nabla \times\left(\mathbf{H}-\mathbf{H}^{I}\right), \xi\right) \\
& +\left(\left(\mathbf{H}-\mathbf{H}^{I}\right)_{t}, \eta\right)+\left(\left(\mathbf{E}-\mathbf{E}^{I}\right), \nabla \times \eta\right) .
\end{aligned}
$$

Note that $\xi \in U^{h}, \eta \in V^{h}, \nabla \times \eta \in U^{h}$. By the interpolation difinition for $\mathbf{E}^{I}$,

$$
\left(\left(\mathbf{E}-\mathbf{E}^{I}\right)_{t}, \xi\right)=0
$$

$$
\left(\mathbf{E}-\mathbf{E}^{I}, \nabla \times \eta\right)=0 .
$$


Then, by (4.2)-(4.4) and Lemmata 3.1 and 3.2,

$$
\frac{1}{2} \frac{d}{d t}\left(\|\xi\|_{0}^{2}+\|\eta\|_{0}^{2}\right)=O\left(h^{k+1}\right)\left(\|\mathbf{H}\|_{k+2}\|\xi\|_{0}+\left\|\mathbf{H}_{t}\right\|_{k+1}\|\eta\|_{0}\right) .
$$

Note that $\xi(0)=\eta(0)=0$. Integrate (4.5) with respect to $t$. Then (4.1) follows by the Schwarz inequality and Gronwell inequalities.

Theorem 4.2. Let $\boldsymbol{E}, \boldsymbol{E}^{h}$ the solutions of (2.6) and (2.8), respectively, and $\mathbf{E}^{I} \in$ $V_{0}^{h}$ the interpolation of $\boldsymbol{E}$. Then

$$
\begin{aligned}
\|\left(\mathbf{E}^{h}\right. & \left.-\mathbf{E}^{I}\right)_{t}\left\|_{0}+\right\| \nabla \times\left(\mathbf{E}^{h}-\mathbf{E}^{I}\right) \|_{0} \\
& =O\left(h^{k+1}\right)\left(\|\mathbf{E}\|_{k+2}+\left(\int_{0}^{t}\left(\left\|\mathbf{E}_{t t}\right\|_{k+1}^{2}+\left\|\mathbf{E}_{t}\right\|_{k+2}^{2}\right) d s\right)^{\frac{1}{2}}\right) .
\end{aligned}
$$

Proof. Let $\xi=\mathbf{E}^{h}-\mathbf{E}^{I}$. Then, by (2.6) and (2.8),

$$
\begin{gathered}
\frac{1}{2} \frac{d}{d t}\left(\left\|\xi_{t}\right\|_{0}^{2}+\|\nabla \times \xi\|_{0}^{2}\right)=\left(\xi_{t t}, \xi_{t}\right)+\left(\nabla \times \xi, \nabla \times \xi_{t}\right) \\
=\left(\left(\mathbf{E}-\mathbf{E}^{I}\right)_{t t}, \xi_{t}\right)+\left(\nabla \times\left(\mathbf{E}-\mathbf{E}^{I}\right), \nabla \times \xi_{t}\right)
\end{gathered}
$$

Because $\xi(0)=\xi_{t}(0)=0$, integrating (4.7) for $t$ and integrating by parts, we can prove that

$$
\begin{aligned}
& \left\|\xi_{t}\right\|_{0}^{2}+\|\nabla \times \xi\|_{0}^{2}=2 \int_{0}^{t}\left(\left(\mathbf{E}-\mathbf{E}^{I}\right)_{t t}, \xi_{t}\right) d s \\
& \quad+2\left(\nabla \times\left(\mathbf{E}-\mathbf{E}^{I}\right), \nabla \times \xi\right)-2 \int_{0}^{t}\left(\nabla \times\left(\mathbf{E}-\mathbf{E}^{I}\right)_{t}, \nabla \times \xi\right) d s .
\end{aligned}
$$

Note that $\xi \in V^{h}, \nabla \times \xi \in U^{h}$. Then by Lemmata 3.1 and 3.2 and the Schwarz inequality,

$$
\begin{aligned}
& \left\|\xi_{t}\right\|_{0}^{2}+\|\nabla \times \xi\|_{0}^{2}=O\left(h^{2 k+2}\right) \int_{0}^{t}\left(\left\|\mathbf{E}_{t t}\right\|_{k+1}^{2}+\left\|\mathbf{E}_{t}\right\|_{k+2}^{2}\right) d s \\
& \quad+\int_{0}^{t}\left(\left\|\xi_{t}\right\|_{0}^{2}+\|\nabla \times \xi\|_{0}^{2}\right) d s+O\left(h^{2 k+2}\right)\|\mathbf{E}\|_{k+2}^{2}+\frac{1}{2}\|\nabla \times \xi\|_{0}^{2} .
\end{aligned}
$$

Hence, (4.6) follows by the Gronwell inequality.

Thus we have achieved the superclose estimates

$$
\begin{aligned}
& \left\|\mathbf{E}^{h}-\mathbf{E}^{I}\right\|_{0}+\left\|\mathbf{H}^{h}-\mathbf{H}^{I}\right\|_{0}=O\left(h^{k+1}\right), \\
& \left\|\left(\mathbf{E}^{h}-\mathbf{E}^{I}\right)_{t}\right\|_{0}+\left\|\nabla \times\left(\mathbf{E}^{h}-\mathbf{E}^{I}\right)\right\|_{0}=O\left(h^{k+1}\right) .
\end{aligned}
$$

\section{Global superconvergence}

In order to achieve global superconvergence, we now define a postprocess operator $\Pi_{2 h}$ as follows.

For the first component of $\mathbf{w} \in U^{h}$, define $\left.\Pi_{2 h} w_{1}\right|_{\hat{e}} \in Q_{k, 2 k-1,2 k-1}(\hat{e})$ such that

$$
\int_{e_{i}}\left(\Pi_{2 h} w_{1}-w_{1}\right) q=0, \quad \forall q \in Q_{k, k-1, k-1}\left(e_{i}\right), \quad i=1, \cdots, 4,
$$

where $\hat{e}=\bigcup_{i=1}^{4} e_{i}$ (see Figure 2). In the similar way, we can define the interpolation postprocess operator $\Pi_{2 h}$ for the second and the third components of $\mathbf{w} \in U^{h}$. 


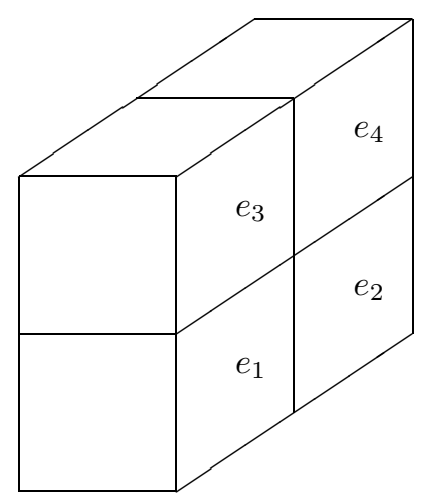

Figure 2

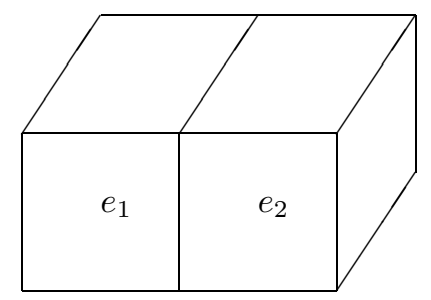

Figure 3

For the first component of $\mathbf{v} \in V^{h}$, define $\left.\Pi_{2 h} v_{1}\right|_{\check{e}} \in Q_{2 k-1, k, k}(\check{e})$ such that

$$
\begin{gathered}
\int_{l_{i}}\left(\Pi_{2 h} v_{1}-v_{1}\right) q d x=0, \quad \forall q \in P_{k-1}\left(l_{i}\right), \quad i=1, \cdots, 8, \\
\int_{\tau_{i}}\left(\Pi_{2 h} v_{1}-v_{1}\right) q d x d y=0, \quad \forall q \in Q_{k-1, k-2}\left(\tau_{i}\right), \quad i=1, \cdots, 4, \\
\int_{\tau_{j}}\left(\Pi_{2 h} v_{1}-v_{1}\right) q d x d z=0, \quad \forall q \in Q_{k-1, k-2}\left(\tau_{j}\right), \quad j=1, \cdots, 4, \\
\int_{e_{i}}\left(\Pi_{2 h} v_{1}-v_{1}\right) q d x d y d z=0, \quad \forall q \in Q_{k-1, k-2, k-2}\left(e_{i}\right), \quad i=1,2,
\end{gathered}
$$

where $\check{e}=e_{1} \bigcup e_{2}$ (see Figure 3 ), $l_{i}$ are edges parallel to the $x$ axis, $\tau_{i}, \tau_{j}$ are surfaces perpendicular to the $z$ axis or $y$ axis, respectively. We can also define $\Pi_{2 h}$ for the second and third components of $\mathbf{v} \in V^{h}$ similarly.

For the above interpolation postprocess operator $\Pi_{2 h}$, it is easy to see that for $\mathbf{w}, \mathbf{v} \in\left[H^{k+1}\right]^{3}$,

$$
\begin{gathered}
\left\|\Pi_{2 h} \mathbf{w}-\mathbf{w}\right\|_{0} \leq C h^{k+1}\|\mathbf{w}\|_{k+1}, \quad\left\|\Pi_{2 h} \mathbf{v}-\mathbf{v}\right\|_{0} \leq C h^{k+1}\|\mathbf{v}\|_{k+1}, \\
\Pi_{2 h} \mathbf{w}=\Pi_{2 h} \mathbf{w}^{I}, \quad \Pi_{2 h} \mathbf{v}=\Pi_{2 h} \mathbf{v}^{I},
\end{gathered}
$$


where $\mathbf{w}^{I} \in U^{h}, \mathbf{v}^{I} \in V^{h}$ are interpolations of $\mathbf{w}$ and $\mathbf{v}$. In addition, for $\mathbf{w} \in U^{h}$, $\mathbf{v} \in V^{h}$

$$
\left\|\Pi_{2 h} \mathbf{w}\right\|_{0} \leq C\|\mathbf{w}\|_{0}, \quad\left\|\Pi_{2 h} \mathbf{v}\right\|_{0} \leq C\|\mathbf{v}\|_{0} .
$$

Based on the above interpolation postprocess operator $\Pi_{2 h}$ and its properties (5.1)-(5.3), we can achieve global superconvergence as follows.

Theorem 5.1. Under the conditions of Theorems 4.1 and 4.2,

$$
\begin{gathered}
\left\|\Pi_{2 h} \mathbf{E}^{h}-\mathbf{E}\right\|_{0}+\left\|\Pi_{2 h} \mathbf{H}^{h}-\mathbf{H}\right\|_{0}=O\left(h^{k+1}\right)\left(\|\mathbf{E}\|_{k+1}+\|\mathbf{H}\|_{k+1}\right. \\
\left.+\left(\int_{0}^{t}\left(\|\mathbf{H}\|_{k+2}^{2}+\left\|\mathbf{H}_{t}\right\|_{k+1}^{2}\right) d s\right)^{\frac{1}{2}}\right),
\end{gathered}
$$

$$
\left\|\Pi_{2 h} \mathbf{E}_{t}^{h}-\mathbf{E}_{t}\right\|_{0}=O\left(h^{k+1}\right)\left(\|\mathbf{E}\|_{k+2}+\left(\int_{0}^{t}\left(\left\|\mathbf{E}_{t t}\right\|_{k+1}^{2}+\left\|\mathbf{E}_{t}\right\|_{k+2}^{2}\right) d s\right)^{\frac{1}{2}}\right) .
$$

Proof. By (5.2)

$$
\Pi_{2 h} \mathbf{E}^{h}-\mathbf{E}=\Pi_{2 h} \mathbf{E}^{h}-\Pi_{2 h} \mathbf{E}^{I}+\Pi_{2 h} \mathbf{E}-\mathbf{E} .
$$

Then, by (5.1), (5.3) and Theorem 4.1,

$$
\begin{aligned}
& \left\|\Pi_{2 h} \mathbf{E}^{h}-\mathbf{E}\right\|_{0} \leq C\left\|\mathbf{E}^{h}-\mathbf{E}^{I}\right\|_{0}+O\left(h^{k+1}\right)\|\mathbf{E}\|_{k+1} \\
& \quad=O\left(h^{k+1}\right)\left(\|\mathbf{E}\|_{k+1}+\left(\int_{0}^{t}\left(\|\mathbf{H}\|_{k+2}^{2}+\left\|\mathbf{H}_{t}\right\|_{k+1}^{2}\right) d s\right)^{\frac{1}{2}}\right) .
\end{aligned}
$$

Similarly,

$$
\left\|\Pi_{2 h} \mathbf{H}^{h}-\mathbf{H}\right\|_{0}=O\left(h^{k+1}\right)\left(\|\mathbf{H}\|_{k+1}+\left(\int_{0}^{t}\left(\|\mathbf{H}\|_{k+2}^{2}+\left\|\mathbf{H}_{t}\right\|_{k+1}^{2}\right) d s\right)^{\frac{1}{2}}\right) .
$$

Hence, (5.4) follows from (5.6) and (5.7). Similarly, (5.5) can be proved from (5.1)(5.3) and Theorem 4.2.

In order to achieve global superconvergence for $\nabla \times \mathbf{E}$ in (2.8), we shall construct the interpolation postprocess operator $\Pi_{3 h}$ with higher order on the larger element. For the first component of $\mathbf{v} \in V^{h}$ we have $\left.\Pi_{3 h} v_{1}\right|_{\tilde{e}} \in Q_{3 k-1,2 k, 2 k}(\tilde{e})$ such that

$$
\begin{array}{lll}
\int_{l_{i}}\left(\Pi_{3 h} v_{1}-v_{1}\right) q d x=0, & \forall q \in P_{k-1}\left(l_{i}\right), & i=1, \cdots, 27, \\
\int_{\tau_{i}}\left(\Pi_{3 h} v_{1}-v_{1}\right) q d x d y=0, & \forall q \in Q_{k-1, k-2}\left(\tau_{i}\right), & i=1, \cdots, 18, \\
\int_{\tau_{j}}\left(\Pi_{3 h} v_{1}-v_{1}\right) q d x d z=0, & \forall q \in Q_{k-1, k-2}\left(\tau_{j}\right), & j=1, \cdots, 18, \\
\int_{e_{i}}\left(\Pi_{3 h} v_{1}-v_{1}\right) q d x d y d z=0, & \forall q \in Q_{k-1, k-2, k-2}\left(e_{i}\right), & i=1, \cdots, 12,
\end{array}
$$

where $\tilde{e}=\bigcup_{i=1}^{12} e_{i}$ (see Figure 4 ), $l_{i}$ are edges parallel to the $x$ axis, $\tau_{i}, \tau_{j}$ are surfaces perpendicular to the $z$ axis or $y$ axis, respectively. $\Pi_{3 h}$ for the second or the third components of $\mathbf{v} \in V^{h}$ can be defined similarly.

For $\Pi_{3 h}$, it is easy to see that (5.2) follows, and

$$
\left\|\Pi_{3 h} \mathbf{v}-\mathbf{v}\right\|_{1}=O\left(h^{k+1}\right)\|\mathbf{v}\|_{k+2},
$$




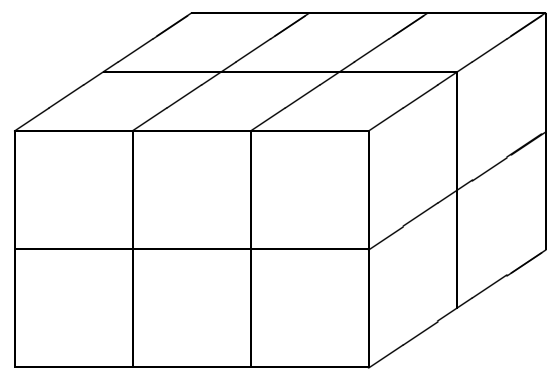

Figure 4

$$
\left\|\nabla \times\left(\Pi_{3 h} \mathbf{v}\right)\right\|_{0} \leq C\|\nabla \times \mathbf{v}\|_{0}, \quad \forall \mathbf{v} \in V^{h} .
$$

Hence, as we did for Theorem 5.1, it is easy to prove that

$$
\left\|\nabla \times\left(\Pi_{3 h} \mathbf{E}^{h}-\mathbf{E}\right)\right\|_{0}=O\left(h^{k+1}\right)\left(\|\mathbf{E}\|_{k+2}+\left(\int_{0}^{t}\left(\left\|\mathbf{E}_{t t}\right\|_{k+1}^{2}+\left\|\mathbf{E}_{t}\right\|_{k+2}^{2}\right) d s\right)^{\frac{1}{2}}\right) .
$$

When $k \geq 2$, then $2 k-1 \geq k+1$ and $Q_{k+1, k+1, k+1} \subset Q_{2 k-1,2 k, 2 k}$. Hence, we can use 8 elements to construct $\tilde{\Pi}_{2 h}$ satisfying (5.2), (5.8), (5.9), and so (5.10).

\section{Generalization}

Let us consider (1.1) with the variable coefficients $\epsilon \in C^{1}(\Omega), \sigma \in C^{1}(\Omega)$ and $\mu \in C^{1}(\Omega)$. Introduce the approximations of $\epsilon, \sigma, \mu$ satisfying

$$
\left.\bar{\epsilon}\right|_{e}=\frac{\int_{e} \epsilon}{|e|},\left.\quad \bar{\sigma}\right|_{e}=\frac{\int_{e} \sigma}{|e|},\left.\quad \bar{\mu}\right|_{e}=\frac{\int_{e} \mu}{|e|},
$$

where $|e|$ is the area of $e$. Then, because $\mathbf{E}^{I}$ is the $L^{2}$-projection of $\mathbf{E}$ on $U^{h}$, it is easy to see that

$$
\begin{aligned}
\left(\epsilon(\mathbf{E}-\mathbf{E})_{t}^{I}, \Phi\right) & =\left((\epsilon-\bar{\epsilon})\left(\mathbf{E}-\mathbf{E}^{I}\right)_{t}, \Phi\right)=O(h)\|\epsilon\|_{1, \infty}\left\|\left(\mathbf{E}-\mathbf{E}^{I}\right)_{t}\right\|_{0}\|\Phi\|_{0} \\
& =O\left(h^{k+1}\right)\left\|\mathbf{E}_{t}\right\|_{k}\|\Phi\|_{0}, \quad \forall \Phi \in U^{h} .
\end{aligned}
$$

Similarly,

$$
\left(\sigma\left(E-E^{I}\right), \Phi\right)=O\left(h^{k+1}\right)\|\mathbf{E}\|_{k}\|\Phi\|_{0}, \quad \forall \Phi \in U^{h} .
$$

By Lemma 3.2, $\forall \Psi \in V^{h}$,

$$
\begin{aligned}
(\mu(\mathbf{H} & \left.\left.-\mathbf{H}^{I}\right)_{t}, \Psi\right) \\
& =\left(\bar{\mu}\left(H-H^{I}\right)_{t}, \Psi\right)+\left((\mu-\bar{\mu})\left(H-H^{I}\right)_{t}, \Psi\right) \\
& =O\left(h^{k+1}\right)\|\|\left(\mathbf{H} \quad-{ }^{I}\right)_{t}\left\|_{0}\right\| \Psi \|_{0} \\
& =O\left(h^{k+1}\right)\left\|\mathbf{H}_{t}\right\|_{k+1}\|\Psi\|_{0} .
\end{aligned}
$$

Hence, as in Theorem 4.1, it can be proved that if $T_{h}$ is a cubic mesh, and $\mathbf{E}, \mathbf{H}$ are smooth enough, then

$$
\left\|\mathbf{E}^{h}-\mathbf{E}^{I}\right\|_{0, \epsilon}+\left\|\mathbf{H}^{h}-\mathbf{H}^{I}\right\|_{0, \mu}=O\left(h^{k+1}\right),
$$


where

$$
\|w\|_{0, g}=\left(\int_{\Omega} g w^{2}\right)^{\frac{1}{2}}
$$

$g \geq g_{\min }>0, \mathbf{E}^{h}, \mathbf{H}^{h}$ are the solutions of (2.2) with variable coefficients, $\mathbf{E}^{I} \in U^{h}$, $\mathbf{H}^{I} \in V^{h}$ are the interpolations of $\mathbf{E}$ and $\mathbf{H}, \mathbf{E}, \mathbf{H}$ are the solutions of (1.1). The similar result for the finite element equation (2.8) with the variable coefficients can be proved similarly. Based on the above results, the global supercovergence as in Theorem 5.1 can be achieved.

When $\Omega$ is not a cubic domain, we cannot construct the cubic mesh on $\Omega$. In order to achieve the superconvergence as in Sections 3, 4, and 5, we construct an almost cubic mesh on $\Omega$ as follows.

First, make a cubic domain $\hat{\Omega}$ such that $\Omega \subset \hat{\Omega}$; then construct a cubic mash $\hat{T}_{h}$ on $\hat{\Omega}$. Let

$$
\Omega_{1}=\bigcup_{\hat{e} \subset \Omega} \hat{e}, \quad \Omega_{2}=\bigcup_{\hat{e} \cap \partial \Omega \neq \emptyset} \hat{e}
$$

where $\hat{e}$ is the element of $\hat{T}_{h}$. Let $e$ be $\hat{e}$ in $\Omega_{1}$, and when $\hat{e} \subset \Omega_{2}$, let $\hat{e}$ be divided into two parts:

$$
\hat{e}=e \cup e^{t}, \quad e \cap e^{t}=\emptyset, \quad e \subset \Omega, \quad e^{t} \cap \Omega=\emptyset .
$$

Then the almost cubic mesh is

$$
T_{h}=\left(\bigcup_{e \subset \Omega_{1}} e\right) \cup\left(\bigcup_{e \subset \Omega \backslash \Omega_{1}} e\right)
$$

For the above almost cubic mesh, it is easy to see that $e$ is a cubic element in $\Omega_{1}$ and meas $\left(\Omega \backslash \Omega_{1}\right)=O(h)$. Hence, by $(5.3), \forall \Psi \in V^{h}$,

$$
\begin{aligned}
(\mu(\mathbf{H} & \left.\left.-\mathbf{H}^{I}\right)_{t}, \Psi\right)=\int_{\Omega_{1}} \mu\left(\mathbf{H}-\mathbf{H}^{I}\right)_{t} \Psi+\int_{\Omega \backslash \Omega_{1}} \mu\left(\mathbf{H}-\mathbf{H}^{I}\right)_{t} \Psi \\
& =O\left(h^{k+1}\right)\left\|\mathbf{H}_{t}\right\|_{k}\|\Psi\|_{0}+O(1)\left\|\left(\mathbf{H}-\mathbf{H}^{I}\right)_{t}\right\|_{0, \Omega \backslash \Omega_{1}}\|\Psi\|_{0, \Omega \backslash \Omega_{1}} \\
& =O\left(h^{k+1}\right)\left\|\mathbf{H}_{t}\right\|_{k}\|\Psi\|_{0}+O\left(h^{k}\right)\left\|\mathbf{H}_{t}\right\|_{k, \Omega \backslash \Omega_{1}}\|\Psi\|_{0, \Omega \backslash \Omega_{1}} \\
& =O\left(h^{k+1}\right)\left\|\mathbf{H}_{t}\right\|_{k}\|\Psi\|_{0}+O\left(h^{k}\right)\left\|\mathbf{H}_{t}\right\|_{k, \infty, \Omega \backslash \Omega_{1}}\left(\operatorname{meas}\left(\Omega \backslash \Omega_{1}\right)\right)^{\frac{1}{2}}\|\Psi\|_{0, \Omega \backslash \Omega_{1}} \\
& =O\left(h^{k+\frac{1}{2}}\right)\left\|\mathbf{H}_{t}\right\|_{k, \infty}\|\Psi\|_{0} .
\end{aligned}
$$

Similarly, on the almost cubic meshes,

$$
\left(\nabla \times\left(\mathbf{H}-\mathbf{H}^{I}\right), \Phi\right)=O\left(h^{k+\frac{1}{2}}\right)\|\Phi\|_{0}, \quad \forall \Phi \in U^{h} .
$$

And, as in (6.1),

$$
\begin{array}{ll}
\left(\epsilon\left(\mathbf{E}-\mathbf{E}^{I}\right)_{t}, \Phi\right)=O\left(h^{k+1}\right)\left\|\mathbf{E}_{\mathbf{t}}\right\|_{k}\|\Phi\|_{0}, & \forall \Phi \in U^{h}, \\
\left(\sigma\left(\mathbf{E}-\mathbf{E}^{I}\right), \Phi\right)=O\left(h^{k+1}\right)\|\mathbf{E}\|_{k}\|\Phi\|_{0}, & \forall \Phi \in U^{h}, \\
\left(\mathbf{E}-\mathbf{E}^{I}, \nabla \times \Psi\right)=0, & \forall \Psi \in V^{h} .
\end{array}
$$

Then, it can be proved that on the almost cubic meshes,

$$
\left\|\mathbf{E}^{h}-\mathbf{E}^{I}\right\|_{0, \epsilon}+\left\|\mathbf{H}^{h}-\mathbf{H}^{I}\right\|_{0, \mu}=O\left(h^{k+\frac{1}{2}}\right),
$$

where $\mathbf{E}^{h}, \mathbf{H}^{h}$ are the solutions of $(2.2), \mathbf{E}^{I}, \mathbf{H}^{I}$ are the interpolations of $\mathbf{E}, \mathbf{H}, \mathbf{E}$ and $\mathbf{H}$ are the solutions of (2.1). Then, global superconvergence with order $O\left(h^{k+\frac{1}{2}}\right)$ can be achieved on the almost cubic meshes. Similar results can be achieved for the finite element equation (2.8). 


\section{ACKNOWLEDGMENTS}

We would like to thank Dr. J. Zou of Hong Kong Chinese University for his useful discussions. We also would like to thank the referee for helpful suggestions which have been accepted in our paper.

\section{REFERENCES}

[1] P. G. Ciarlet, The Finite Element Method for Elliptic Problems, North-Holland Publishing Company, 1978. MR 58:25001

[2] G. Duvaut, J. Lions, Inequalities in Mechanics and Physics, Springer-Verlag, New York, 1976. MR 58:25191

[3] R. Ewing, R. Lazarov, J. Wang, Superconvergence of the velocity along the Gauss lines in mixed finite element methods, SIAM J. Numer. Anal., 28(1991), pp 1015-1029. MR 92e: 65149

[4] Q. Lin, N. Yan, Superconvergence of mixed finite element methods for Maxwell's equations, Gongcheng Shuxue Xuebao 13(1996), pp 1-10 (in Chinese). MR 98c:65186

[5] Q. Lin, N. Yan, The Construction and Analysis of High Efficiency Finite Element Methods, Hebei University Publishers, 1996.

[6] Q. Lin, N. Yan, A. Zhou, A rectangle test for interpolated finite elements, Proc. of Sys. Sci. E Sys. Engrg., Great Wall (H. K.) Culture Publish Co., 1991, pp 217-229.

[7] P. Monk, A mixed method for approximating Maxwell's equations, SIAM J. Numer. Anal., 28(1991), pp 1610-1634. MR 92j:65173

[8] P. Monk, Analysis of a finite element method for Maxwell's equations, SIAM J. Numer. Anal., 29(1992), pp 714-729. MR 93k:65096

[9] P. Monk, A comparison of three mixed methods for the time-dependent Maxwell's equations, SIAM J. Sci. Stat. Comput., 13(1992), pp 1097-1122. MR 93j:65184

[10] P. Monk, An analysis of Nédélec's method for the special discretization of Maxwell's equations, J. Comput. Appl. Math., 47(1993), pp 101-121. MR 94g:65105

[11] P. Monk, Superconvergence of finite element approximations to Maxwell's equations, $\mathrm{Nu}$ merical Methods for Partial Differential Equations, 10(1994), pp 793-812. MR 95h:65090

[12] J. Nédélec, Mixed finite element in $\mathrm{R}^{3}$, Numer. Math., 35(1980), pp 315-341. MR 81k:65125

[13] A.H.Schatz, I.H.Sloan, L.B.Wahlbin, Superconvergence in finite element methods and meshes which are locally symmetric with respect to a point, SIAM J. Numer. Anal., 33(1996), pp 505-521. MR 98f:65112

[14] L. B. Wahlbin, Superconvergence in Galerkin Finite Element Methods, Springer Lecture Notes in Mathematics, 1605, 1995. MR 98j:65083

Institute of Systems Science, Academia Sinica, Beijing, China

E-mail address: glin@bamboo.iss.ac.cn

E-mail address: yan@bamboo.iss.ac.cn 